\title{
Solar-Supercapacitor Harvesting System Design for Energy-Aware Applications
}

\author{
Moeen Hassanalieragh, Tolga Soyata, Andrew Nadeau, Gaurav Sharma \\ University of Rochester, Dept. of Electrical and Computer Engineering, Rochester, NY 14627 \\ \{m.hassanalieragh,tolga.soyata,andrew.nadeau,gaurav.sharma\}@ rochester.edu
}

\begin{abstract}
Supercapacitors are an emerging choice for energy buffering in field systems and their use in solar-powered field systems has been the focus of recent research. Supercapacitors offer advantages compared to rechargeable batteries for energy buffering due to their energy charge/discharge efficiency as well as environmental friendliness. Additionally, a supercapacitorbased system permits an energy-aware operation due to its superior energy-predictability. This paper describes a circuit for solar/supercapacitor energy harvesting, which includes power and voltage measurements, voltage regulation circuit and RS232 communication capability with the host embedded processor. A complete system is prototyped and its operation is discussed in terms of design parameters.
\end{abstract}

\section{INTRODUCTION}

Recent research focused on powering Wireless Sensor Networks [1] and field systems using supercapacitors [2] due to their potential in providing a batteryless power supply for embedded systems, thereby yielding a much longer operational lifetime. Although these two type of systems do not take advantage of one of the most important high power density feature of supercapacitors, which is the backbone of other high-powered applications such as industrial elevators or electric vehicles [3]-[5], another equally important feature of the supercapacitors are taken advantage of by both of these applications : energy efficiency and their superior energy predictability by observing the supercapacitor terminal voltage, $V_{S C}$ and predicting the remaining energy as $E=\frac{1}{2} C V_{\mathrm{SC}}^{2}$.

Although a rich body of circuit references exist for the building blocks of an energy harvesting system, a complete harvesting system design for incorporating energy awareness into higher system levels is not readily available which details relevant design and runtime issues. In this paper, we introduce a microcontroller-based energy harvester design which receives its energy from multiple solar panels, harvests energy by using a DC-DC converter and stores the harvested energy in two blocks of supercapacitor. This battery-less harvester is intended to power field systems with an embedded CPU, such as Nexus 7, with a target overall system power consumption of $0.5-10 \mathrm{~W}$. We demonstrate results on a prototype we built, shown in Figure 1.

The primary goal of our design is to create a solar harvesting platform that not only buffers energy to sustain operation, but provides the embedded processor with enough information to make intelligent decisions to take advantage of remaining energy. We detail multiple design issues relevant to such a field system harvester and elaborate on each issue.

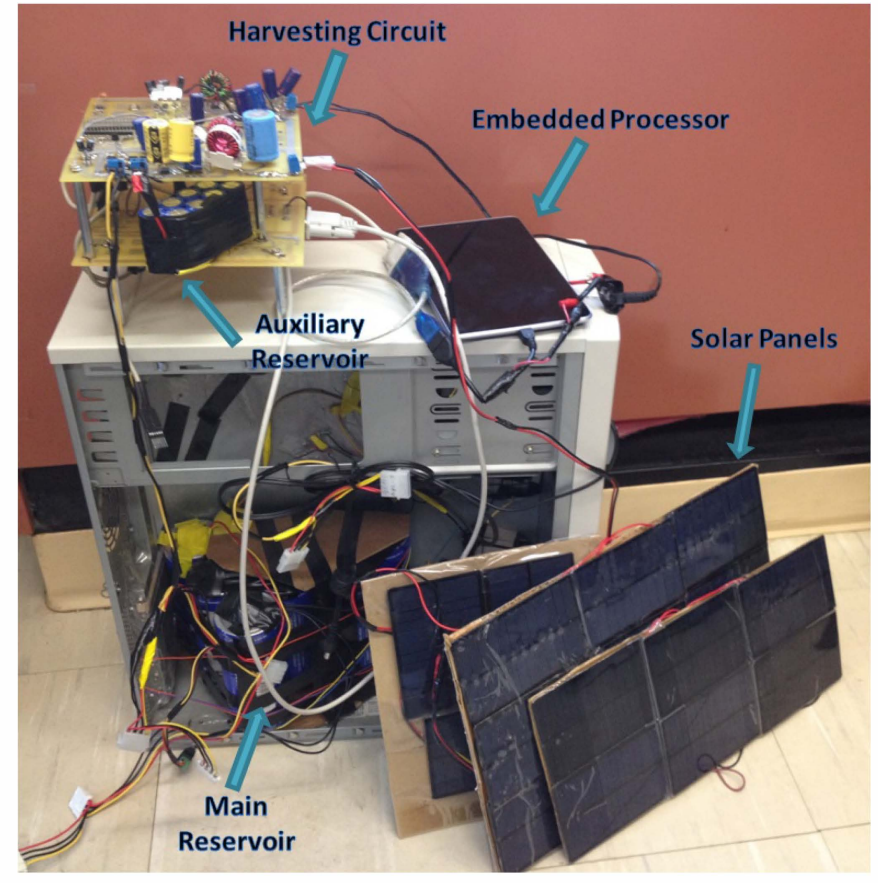

Fig. 1. Our prototype solar/supercapacitor harvesting system along with supercapacitor reservoirs and the embedded processor.

This paper is organized as follows: In Section II, we provide background information on solar panels and supercapacitors. Section III is where we introduce our proposed system, in Section IV details of the harvesting circuit is presented followed by evaluations on our prototype in Section V. We draw conclusions in Section VI.

\section{BACKGROUND AND RELATED WORK}

\section{A. Solar Energy}

In recent years photovoltaic (PV) cells have gained much interest to increase the autonomy of embedded systems. The output characteristics of a PV cell varies non-linearly with environmental conditions such as temperature and irradiation [6], [7]. Also, the power gained from a PV cell greatly depends on its operating point, i.e we need to keep it at its optimum operating point by demanding sufficient amount of energy. When the demanded current from a PV cell is high, its terminal voltage drops to a very small value. This current is denoted by the short circuit current $I_{\text {Solar }}=I_{S C}$. When there is no current demand from the PV cell, its terminal voltage increases to the open circuit voltage. At both of these extreme cases no 

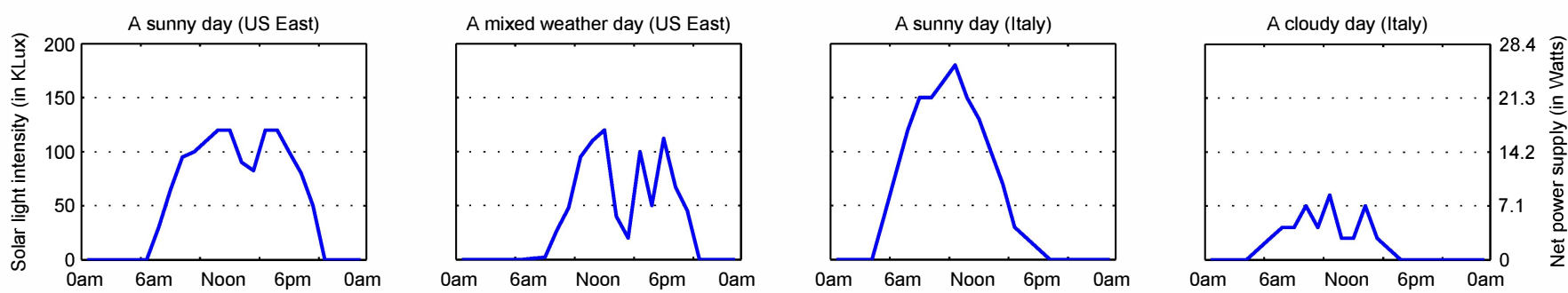

Fig. 2. Example solar power levels under varying weather conditions. Results in Italy obtained from [6]

power is generated by the PV cell. Thus, there is a need for an algorithm which keeps the PV cell at its maximum power point (MPP). These methods are called maximum power point tracking (MPPT) methods. Several MPPT methods have already been proposed [8], [9]. These methods differ in their means of implementation. Based on their level of complexity, some of them need a digital processor to be implemented while the others can be realized using analog components.

We provide solar power into our system using Radio Shack Model 277052 solar panels. These panels have a $6 \mathrm{~V}$ output voltage and can provide $1.5 \mathrm{~W}$ of output power, as per specifications. Having a hybrid architecture of them in parallel and series could yield to desirable operating voltage and power to provide power for a variety of compute-intensive field applications [10], [11].

The harvested power is almost linearly dependent on the solar irradiation levels [12], measured in KLux (Kilo Lumens per $m^{2}$ ). From measurements, we determined that, the specified $1.5 \mathrm{~W}$ solar power for the Radio Shack panels are reached at a $200 \mathrm{KLux}$ sunlight. This is achieved on a very sunny day under bright sunshine. Solar energy patterns for different weather conditions are shown in Figure 2 for a block of 21 panels, organized as a $7 \times 3$ grid. Figure 2 depicts the dependence of solar power output on sunshine patterns. A small cloud block can reduce the solar intensity from 200 KLux to 40 KLux almost instantenously. Our experiments in US East are shown to yield similar patterns to studies in Italy [6]. This emphasizes that, a solar harvesting system must employ an MPPT algorithm by tracking the sunlight patterns continuously to provide efficient results [1].

\section{B. Supercapacitors}

Supercapacitors store energy based on two phenomena occurring at the electrode-electrolyte interface: a) EDL (electric double layer) and b) pseudocapacitance. EDL allows supercapacitors to store energy by layers of charged ions from the electrolyte attracted to the electrode surface by electrostatic forces or selective adsorption of ions into the surface, whereas Pseudocapacitance allows supercapacitors to store energy chemically, similar to batteries [13]. Compared to conventional capacitors, supercapacitors have significantly different operational characteristics which must be considered when a system is designed with them [14]-[16].

Figure 3 shows a set of eight Maxwell $3000 \mathrm{~F}$ supercapacitors [17] connected in series, with individual maximum

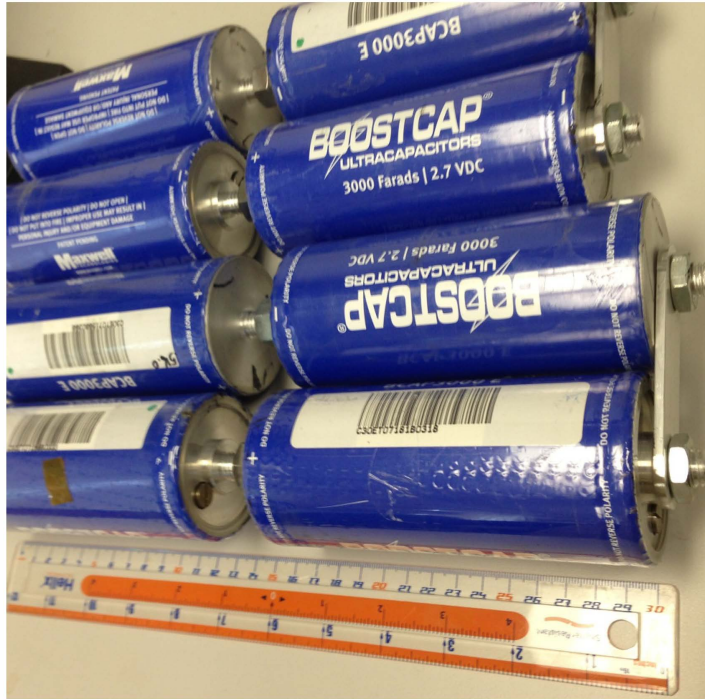

Fig. 3. Energy buffer: 8 series Maxwell BCAP3000 supercapacitors [17].

voltages of $2.7 \mathrm{~V}$ and a combined supercapacitor block voltage of $21.6 \mathrm{~V}$. A total energy of $E=\frac{1}{2} \times 3000 \times 2.7^{2}=10935 \mathrm{~J}$ is stored in each supercapacitor, with a combined storage of $87840 \mathrm{~J}$ total for a block of eight supercapacitors. Note that, this energy estimation can yield erroneous results in certain conditions due to charge redistribution [18]. Due to their capability to store such a high amount of energy, extreme care must be taken to prevent short-circuiting them.

\section{Solar Energy Harvesting System Design}

Figure 4 shows the overall system architecture. Solar energy is buffered on two supercapacitor reservoirs using an energy harvesting circuit. Primary reservoir is intended to power up the embedded processor. Secondary reservoir has the role of supplying energy for the microcontroller that is the crucial part in our energy harvesting circuit. Energy transfer from reservoirs to microcontroller and the embedded processor is realized using two DC-DC converters.

\section{A. Nexus 7 Tablet - Embedded Processor}

Data processing in the field requires substantial computational capability that urges us to use an embedded processor. We have selected Google Nexus 7 tablet which contains Tegra3 quad-core mobile processor. Nexus 7 power consumption varies between $0.5-6 \mathrm{~W}$, based on its frequency and number of active cores. Having extra peripherals attached to Nexus 


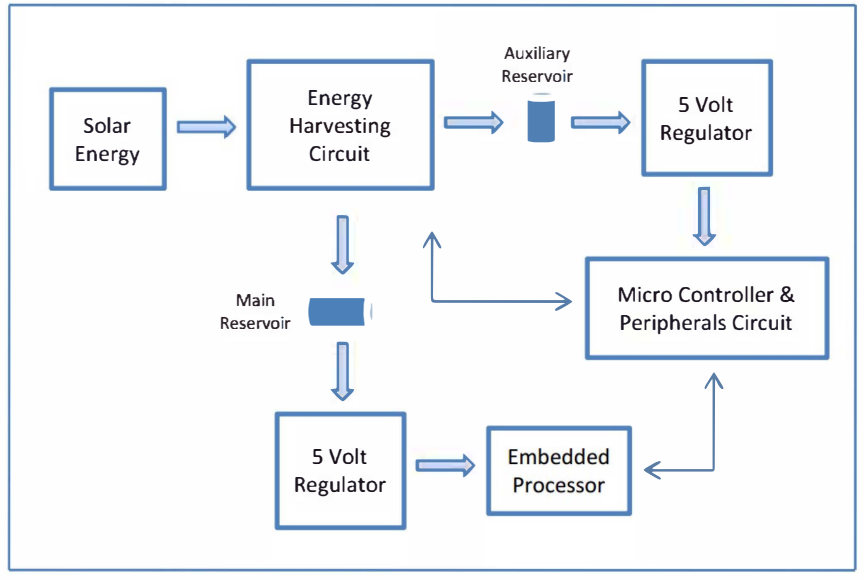

Fig. 4. Architectural diagram of the harvesting system.

7 could lead to a maximum power consumption of $10 \mathrm{~W}$ at $5 \mathrm{~V}$. Nexus 7 power proportionality makes it an ideal choice for field systems that need to adapt the processing power consumption based on the available energy.

\section{B. Microcontroller Selection}

The microcontroller suitable for our application should include the following features: 1) I/O pins to control the MOSFETs. 2) Having an I/O voltage of at least $5 \mathrm{~V}$ in order to be able to turn on/off the MOSFETs. 3) Multiple ADC channels to sample the voltage on different nodes (both differential and single ended). 4) a UART module for transferring information through the RS232 port. The 8-bit PIC16F1783 microcontroller satisfies all of these conditions, and contains an internal voltage reference of $4096 \mathrm{mV}$. An industry standard TTL to RS232 converter ADM232LAN allows connection to a standard RS-232 port, and a gate driver MAX627 is used to drive power MOSFETs directly from PIC I/O pins. The current sense amplifier is MAX4372H allows us to convert 1$2000 \mathrm{~mA}$ current levels to PIC-readable voltage levels, upto $4096 \mathrm{mV}$. Total power consumption of the microcontroller and its peripherals does not exceed $250 \mathrm{~mW}$.

\section{Dual-Reservoir Architecture}

Our system consists of two reservoirs for buffering the harvested energy: The Main Reservoir and the Auxiliary Reservoir are constructed from $8 \times(3000 \mathrm{~F}, 2.7 \mathrm{~V})$ and 9x(50F, $2.7 \mathrm{~V})$ supercapacitors. The Main reservoir provides power to the Nexus 7 tablet, while the Auxiliary reservoir powers the microcontroller and its perhiherals. Having a separate reservoir for the microcontroller ensures that, in case of a complete depletion of the Main Reservoir, the microcontroller and its peripherals could be brought back in operational mode quickly, thereby eliminating the possibility of a deadlocked operational mode.

Energy is channelled to these separate reservoirs through a software switch. Having a wide voltage range on the main reservoir is crucial to take advantage of the maximum available energy in the supercapacitors. Based on Nexus 7 power specifications, we chose LTC1624 as the switching regulator,

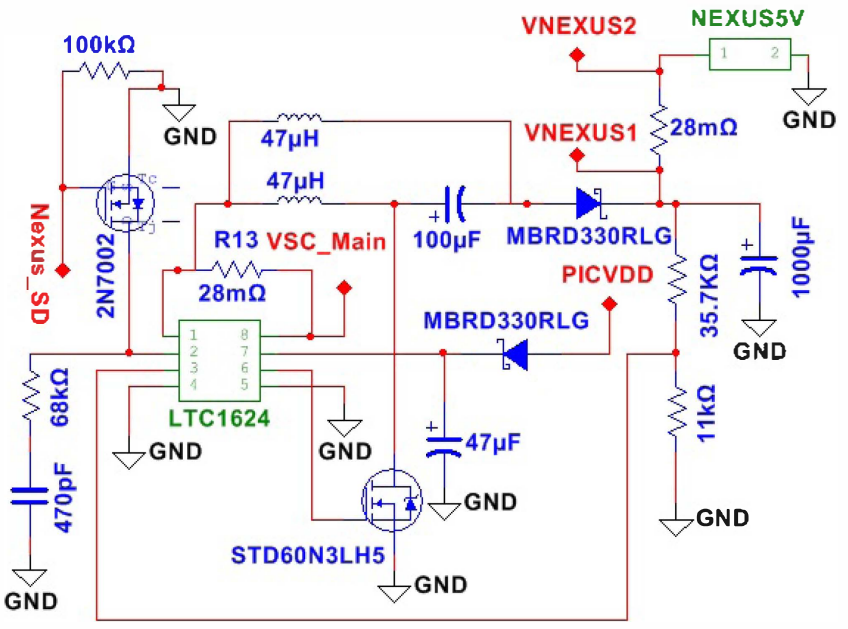

Fig. 5. Nexus 7 Voltage Regulator, input $=(3.5-21.6 \mathrm{~V})$, Output=5 V, $2 \mathrm{~A}$

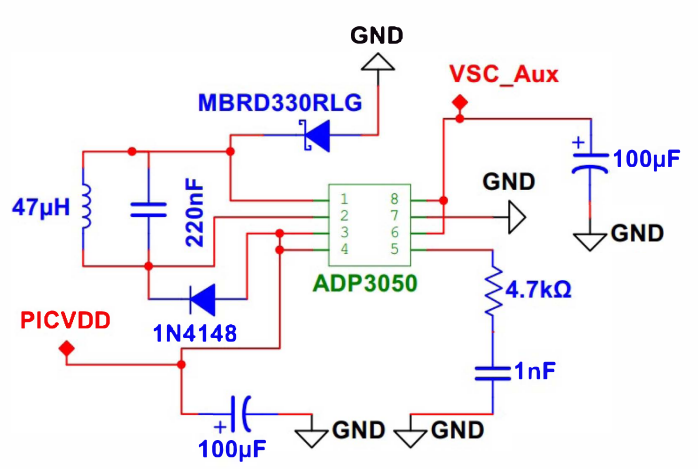

Fig. 6. $\mu \mathrm{C}$ Voltage Regulator, input $=(5.5-24.3 \mathrm{~V})$, Output $=5 \mathrm{~V}, 250 \mathrm{~mA}$

configured in SEPIC mode. Its input voltage range is $3.5-36 \mathrm{~V}$, which completely suits our application. Since this regulator uses an external MOSFET switch, we can provide sufficient power to Nexus 7 via a power MOSFET. Use of this regulator eliminates the need of a gate driver to drive the switch, since it has an internal one. We can see the configuration in Figure 5. This regulator has also the capability to shut down when needed by setting its RUN pin to GND (pin 2). This pin is controlled by the PIC software (Nexus_SD pin) through the 2N7002 transistor. Based on the microcontroller power requirement, we chose ADP3050-5 which is a $5 \mathrm{~V}, 1 \mathrm{~A}$ Buck regulator with an internal switch. The configuration is depicted in Figure 6. Using a Buck regulator imposes the constraint that voltage on the auxiliary reservoir should not go below $5.5 \mathrm{~V}$ for proper operation. $5 \mathrm{~V}$ generated by this regulator also feeds the Boost pin of Nexus regulator thorough a diode. Boost pin actually supplies the voltage of the internal gate driver of LTC1624. This regulator has an internal $5 \mathrm{~V}$ generator for providing the boost voltage, but it only works when the input voltage to the regulator is higher than $5 \mathrm{~V}$. Externally connected $5 \mathrm{~V}$ insures proper operation for voltage range of $3.5-5 \mathrm{~V}$. This block provides sufficient power to a host system running sophisticated energy-aware algorithms [19]-[21]. 


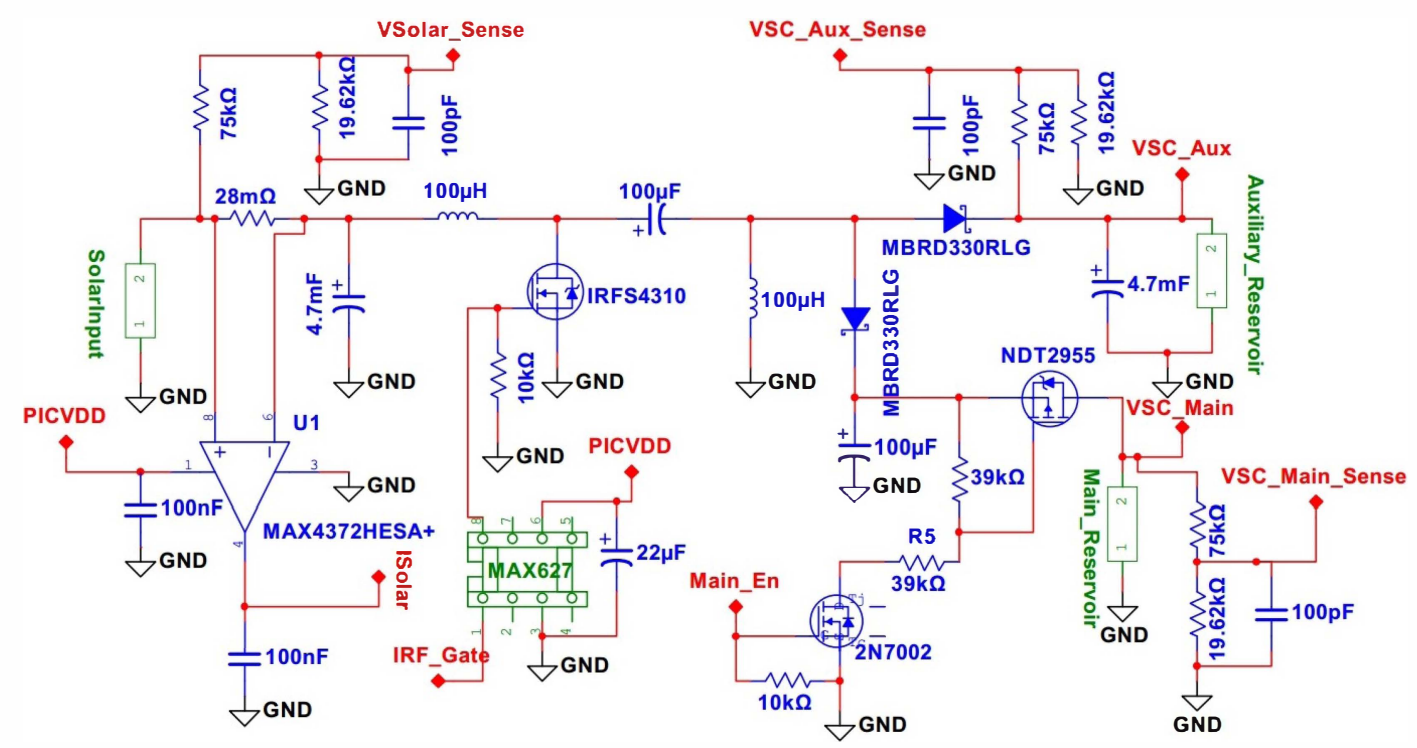

Fig. 7. Solar Energy Harvesting Circuit built around a SEPIC DC-DC converter

\section{Energy Harvesting Circuit}

An energy harvesting circuit is essential to deliver the available input solar energy to supercapacitors. DC-DC converters are a specific type of energy transfer circuits that adjust the amount of energy taken from the input in order to keep their output voltage level at a certain value. It is essential to notice that in the harvesting platform we do not intend to go from one voltage level to another, but keep the input solar panels voltage at the optimum point and transfer the harvested energy to reservoirs. We will go through the harvesting circuit elements in detail in following subsections.

\section{A. SEPIC DC-DC Converter}

In this paper we have used a special class of DC-DC converters as the energy transfer mechanism. While the most commonly used DC-DC converter designs are Buck, Boost, or Buck-Boost [22], our experiments show that, a less common design, SEPIC Converter [23] is lot more suitable due to the following reasons:

1) While Buck and Boost designs can only up-convert and down-convert, respectively [22], SEPIC can be used as the energy transfer element in a variety of input - output voltage ranges.

2) SEPIC has a continuous-input current and discontinuous-output current draw [23]. With proper electrolytic capacitors on the output side of the system, capacity losses at the supercapacitors at high frequencies [24] can be eliminated. Therefore, SEPIC is a reasonably efficient solution for a an energy transfer circuit that is providing current into a supercapacitor.

3) SEPIC provides a graceful short-circuit response [23] which is important for field systems working in harsh environments.

Figure 7 shows the harvesting circuit. As mentioned in section II, we need to keep the solar panel input power at the optimum point in order to absorb the maximum power.
In SEPIC configuration this is achieved by adjusting the duty cycle of the switch based on the feedback from measuring the voltage of the solar panel. As the duty cycle of the switch control signal increases, the leftmost inductor in SEPIC configuration is turned on more percentage of time and this means increased average current demand from the solar panel and vice versa. Thus, we can move in I-V curve of the solar panel by adjusting the duty cycle of the switch signal. This signal is denoted by IRF_Gate in Figure 7 which is generated by the microcontroller.

\section{B. Microcontroller Software}

An important issue in solar harvesting platforms is finding the maximum power point of the solar panel. The microcontroller software is responsible for this task. We used a periodic calibration technique in which the system tries to find the new optimum point by searching the points in a region around the current optimum point in fixed intervals. The optimum point is simply found by sampling the input current and voltage in search space and find the one with maximum product. This algorithm is less prone to getting stuck in local maxima compared to gradient descent algorithms if the search space is large enough around the current optimum point. The calibration period is chosen to be five minutes based on the fact the rate of change in environmental conditions such as light intensity, temperature, etc. is not very high. The value of input capacitance in SEPIC plays an important role in our search based algorithm. The higher its value the slower the change is in input I-V curve. It must be high enough so that the microcontroller ADC sampling rate yields to sufficient search points for finding the optimum point.

\section{Power MOSFET Selection and Gate Driver}

The transistor switch in the SEPIC converter plays an important role in the harvesting circuit functionality and its efficiency. When choosing a transistor switch several parameters must be taken into account. The threshold voltage, ON 
resistance, maximum drain source voltage in off state and overall gate charge. In SEPIC configuration there can be a maximum voltage of Vin + Vout over the transistor at the off state, so this should be strictly taken into consideration. ON resistance of the switch has a substantial effect on efficiency of the circuit. ON resistance is dependent on the gate source voltage with which the transistor is driven. Overall gate charge of the transistor determines how long it takes for the transistor to change state from $\mathrm{ON}$ to $\mathrm{OFF}$ and vice versa. During this transition, transistor is consuming a considerable amount of energy. In order to provide the gate charge in a smaller amount of time, use of a gate driver is crucial. As shown in Figure 7, MAX627 is used as the gate driver to drive the SEPIC switch.

\section{Current Sensing}

Current sensing is of high importance in harvesting circuits for determining the maximum power point. Basically current sensing is done through measuring the difference voltage across a small resistor which is called the sense resistor. Conventional values for sense resistors range from 5-100 $\mathrm{m} \Omega$ for decreasing power loss on the sense part. Since voltage drop on the sense resistor would be so small, we used a current sense amplifier for amplifying this voltage and then feeding it to ADC of the microcontroller.

An important factor for choosing a current sense amplifier is where the sense resistor is actually placed in the circuit. Sense resistor can be placed either right after the input source (high side sensing) or on the load part (low side sensing). In high side sensing configuration, the current sense amplifier will face a high common mode voltage that must be considered when choosing a current sense amplifier. Based on our requirements, we chose MAX4372H which is a high side current sense amplifier with a gain of 100 . Current sense amplifiers are designed to measure DC current so their effective bandwidth is not high. This must be taken into account when measuring fast varying currents. Output of the current sense amplifier is denoted by ISolar in Figure 7. The value of the sense resistor must be chosen in a way that the current sense amplifier's output will not exceed the maximum sampling voltage of the ADC. We did not use a current sense amplifier for sensing the outgoing current to Nexus 7. As shown in Figure 5, the difference voltage across the sense resistor is directly sampled by microcontroller's differential ADC since they do not have a high common voltage.

\section{Evaluation}

\section{A. Efficiency Analysis}

Power consumption in the harvesting circuit mostly consists of two parts. The first one is the quiescent current of the present integrated circuits and the other one is due to energy loss in circuit components such as transistors and inductors. The first term manifests in the form of constant current loss, so the power consumption will be higher when the input voltage increases.

The harvesting efficiency was determined by measuring the input solar power and the average power harvested on the auxiliary reservoir in a certain amount of time. We chose the auxiliary reservoir for the measurement since it feeds the microcontroller regulation circuit. The experiment was conducted in a wide range of input solar power. The results are shown in Table I. At low solar power input, the quiescent power consumption causes the efficiency to drop. At high solar power input, the circuit components loss (especially the SEPIC switch) dominates and causes the efficiency to decrease.

\begin{tabular}{|c||c|}
\hline Input Power (W) & Efficiency (\%) \\
\hline 1.4 & 77.2 \\
\hline 5.6 & 80.6 \\
\hline 7 & 84.2 \\
\hline 9.8 & 78.3 \\
\hline 12.6 & 75.6 \\
\hline 18.2 & 75.1 \\
\hline 21 & 74.3 \\
\hline 23.8 & 72.6 \\
\hline
\end{tabular}

TABLE I

Measured Harvesting Efficiency at Different Solar Powers

\section{B. Supercapacitor Energy Estimation}

Measuring the remaining energy in a supercapacitor using the formula $\frac{1}{2} C V_{\mathrm{SC}}^{2}$ holds true when it is in a stable condition. Supercapacitors differ from conventional capacitors in a way that they consist of multiple resistance-capacitance branches with different time constants [16]. Measurements are valid only when all of the branches have reached a stable condition and no charge distribution is happening between branches. Thus, if we intend to compute the remaining energy by observing the terminal voltage in non-stable scenarios, we should keep track of branches state of charge. Recently a Kalman filtering approach is introduced to track and estimate the remaining energy in a supercapacitor by observing its terminal voltage and the total current flowing through the supercapacitor [18].

\section{Supercapacitor Overcharging Consideration}

Experiments we conducted on multiple supercapacitors showed manufacturing tolerances ranging from $2855 \mathrm{~F}$ to $3139 \mathrm{~F}$ for Maxwell $3000 \mathrm{~F}$ supercapacitors [17]. Although the average is almost a perfect $3039 \mathrm{~F}$, there is a subtle problem. If the block of eight supercapacitors are operated at $21.6 \mathrm{~V}$, the same current flowing through them will charge the smallest supercapacitor to a higher voltage, thereby yielding a $2.87 \mathrm{~V}$ on the smallest (i.e., $2855 \mathrm{~F}$ ), and a $2.61 \mathrm{~V}$ on the largest supercapacitor (i.e., $3139 \mathrm{~F}$ ). In other words, while the smallest supercapacitor is overcharged, the largest one is underchanged. While sophisticated circuit techniques can be employed to individually control the supercapacitor voltages in the block, the simplest solution is to limit the supercapacitor block voltage $\left(V_{S C}\right)$ to a $V_{S C \max }<21.6 \mathrm{~V}$, where the smallest supercapacitor stays below $2.7 \mathrm{~V}$. This ends up being $V_{S C \max }=20.3 \mathrm{~V}$.

\section{Connection to Nexus 7}

Nexus 7 tablet primarily uses an internal Li-Ion battery. In order to power up the tablet using the custom regulator, we had 
to remove the battery and manually connect the supply pins. Li-Ion battery block has extra connections which report state of charge of the battery to the hardware. The lack of existence of these connections caused the operating system to assume that the battery is out of power and it automatically turned off the power to the USB port. The USB port is connected to RS232 connection of the microcontroller through USB-RS232 converter. We connected the $5 \mathrm{~V}$ feeding the tablet directly to supply pins of the USB port to solve the problem.

\section{CONCLUSIONS AND FUTURE WORK}

This paper introduces a SEPIC-based solar energy harvester, which buffers the harvested energy in two supercapacitor blocks. Our proof-of-concept circuit is capable of harvesting solar energy from 18 solar panels (a total of $24 \mathrm{~W}$ at $200 \mathrm{KLux}$ solar intensity). The harvested solar energy is intended to power up an embedded processor for conducting data-intensive field processing. Continuous power measurements inside the circuit and reporting it to the embedded processor allows the software to make energy-aware decisions.

Although the main reservoir block can theoretically store $\approx 88 \mathrm{KJ}$ energy, in practice, we found a batch of Maxwell $3000 \mathrm{~F}$ supercapacitors to have values in the $2855 \mathrm{~F}$ to $3139 \mathrm{~F}$ range. This implies overcharging on the smallest one, if the block of eight supercapacitors are charged to $8 \times 2.7=21.6 \mathrm{~V}$. Therefore, we suggest the simplest method of keeping the block voltage at $20.3 \mathrm{~V}$ which will eliminate the overcharging issue, albeit at the expense of leaving unused energy in the block. Another solution to overcharging is the parallel supercapacitor configuration, which we leave as future work.

\section{ACKNOWLEDGMENT}

This work was supported in part by the National Science Foundation grant CNS-1239423 and a gift from Nvidia Corp.

\section{REFERENCES}

[1] Davide Brunelli, Luca Benini, Clemens Moser, and Lothar Thiele, "An efficient solar energy harvester for wireless sensor nodes," in Conf. on Design, Automation and Test in Europe (DATE), Munich, Germany, Mar. 2008, pp. 104-109.

[2] Amal Fahad, Tolga Soyata, Tai Wang, Gaurav Sharma, Wendi Heinzelman, and Kai Shen, "SOLARCAP: super capacitor buffering of solar energy for self-sustainable field systems," in Proceedings of the 25th IEEE International System-on-Chip Conference, Niagara Falls, NY, Sep 2012, pp. 236-241.

[3] N. Jinrui, W. Zhifu, and R. Qinglian, "Simulation and Analysis of Performance of a Pure Electric Vehicle with a Super-capacitor," in IEEE Vehicle Power and Propulsion Conference, 2006, pp. 1-6.

[4] H. Zhang, Y. Sun, S. Ding, and Y. Wang, "Application of super capacitor with full-digital converter in hybrid electric vehicle energy transmission system," in 27th Chinese Control Conference, 2008, pp. 212-215.

[5] Alfred Rufer and Philippe Barrade, "A supercapacitor-based energy storage system for elevators with soft commutated interface," IEEE Transactions on Industry Applications, vol. 38, no. 5, pp. 1151-1159, 2002.

[6] Roberto Faranda and Sonia Leva, "Energy comparison of mppt techniques for pv systems," WSEAS Transactions on Power Systems, vol. 3, no. 6, pp. 446-455, 2008.

[7] Davide Brunelli, Clemens Moser, Lothar Thiele, and Luca Benini, "Design of a solar-harvesting circuit for batteryless embedded systems," Circuits and Systems I: Regular Papers, IEEE Transactions on, vol. 56, no. 11, pp. 2519-2528, 2009.
[8] D.P.Hohm and M.E.Ropp, "Comparative study of maximum power point tracking algorithms using an experimental, programmable, maximum power point tracking test bed," in Proc. Photovoltaic Specialist Conference, 2000, pp. 1699-1702.

[9] D.S. Hyun D.Y. Lee, H.J. Noh and I.Choy, "An improved mppt converter using current compensation method for small scaled pvapplications," in Proc. APEC, 2003, pp. 540-545.

[10] Tolga Soyata, He Ba, Wendi Heinzelman, Minseok Kwon, and Jiye Shi, "Accelerating mobile cloud computing: A survey," in Communication Infrastructures for Cloud Computing, H. T. Mouftah and B. Kantarci, Eds., chapter 8, pp. 175-197. IGI Global, Hershey, PA, USA, Sep 2013.

[11] Tolga Soyata, Rajani Muraleedharan, Colin Funai, Minseok Kwon, and Wendi Heinzelman, "Cloud-Vision: Real-Time face recognition using a Mobile-Cloudlet-Cloud acceleration architecture," in Proceedings of the 17th IEEE Symposium on Computers and Communications (IEEE ISCC 2012), Cappadocia, Turkey, Jul 2012, pp. 59-66.

[12] Dave Freeman, "Introduction to Photovoltaic Systems Maximum Power Point Tracking," http://www.ti.com/lit/an/slva446/slva446.pdf, 2010.

[13] B.E. Conway, V. Birss, and J. Wojtowicz, "The role and utilization of pseudocapacitance for energy storage by supercapacitors," Journal of Power Sources, vol. 66, no. 12, pp. 1 - 14, 1997.

[14] Jianjun Niu, Wendy G. Pell, and Brian E. Conway, "Requirements for performance characterization of $\mathrm{c}$ double-layer supercapacitors: Applications to a high specific-area c-cloth material," Journal of Power Sources, vol. 156, no. 2, pp. 725 - 740, 2006.

[15] S. Buller, E. Karden, D. Kok, and R.W. De Doncker, "Modeling the dynamic behavior of supercapacitors using impedance spectroscopy," in Industry Applications Conference, 2001. Thirty-Sixth IAS Annual Meeting. Conference Record of the 2001 IEEE, 2001, vol. 4, pp. 25002504 vol.4.

[16] L. Zubieta and Richard Bonert, "Characterization of double-layer capacitors for power electronics applications," Industry Applications, IEEE Transactions on, vol. 36, no. 1, pp. 199-205, 2000.

[17] Maxwell Corp., "K2 Series High Capacity Cells," http://www.maxwell. com/products/ultracapacitors/products/k2-series, 2012.

[18] Andrew Nadeau, Gaurav Sharma, and Tolga Soyata, "State-of-charge estimation for supercapacitors: A kalman filtering formulation," in Proceedings of the 2014 IEEE International Conference on Acoustics, Speech and Signal Processing (ICASSP 2013), Florence, Italy, May 2014, pp. 2213-2217.

[19] Tolga Soyata and Eby G. Friedman, "Retiming with non-zero clock skew, variable register and interconnect delay," in Proceedings of the IEEE Conference on Computer-Aided Design, Nov 1994, pp. 234-241.

[20] Tolga Soyata and Eby G. Friedman, "Synchronous performance and reliability improvements in pipelined asics," in Proceedings of the IEEE ASIC Conference, Sep 1994, pp. 383-390.

[21] Tolga Soyata, Eby G. Friedman, and J. H. Mulligan, "Integration of clock skew and register delays into a retiming algorithm," in Proceedings of the International Symposium on Circuits and Systems, May 1993, pp. 1483-1486.

[22] Abraham Pressman, Keith Billings, and Taylor Morey, Switching Power Supply Design, Mc Graw-Hill, 2009.

[23] Texas Instruments Incorporated, "Designing DC/DC converters based on SEPIC technology," http://www.ti.com/lit/an/slyt309/slyt309.pdf, 2013.

[24] Maxwell Corp., "Maxwell BOOSTCAP Product Guide," http://www.maxwell.com/products/ultracapacitors/docs/1014627 \_ boostcap\_product\_guide.pdf, 2013. 